\title{
An effect of speed on induced motion
}

\author{
HANS WALLACH and ROBERT BECKLEN \\ Swarthmore College, Swarthmore, Pennsylvania
}

\begin{abstract}
Reciprocating horizontal motion of a pattern of vertical lines caused horizontal induced motion of a dot that underwent vertical reciprocating motion. The real vertical motion and the induced horizontal motion of the dot resulted in a circular or oval apparent path. Increasing the two motion speeds caused the horizontal component of this resultant path to become smaller, a change that indicated a diminished induced effect. This effect of high motion speed was not due to a blurring of the moving line pattern at high speeds, since strong objective blurring of the line pattern did not diminish the extent of the induced motion it caused. When the eyes pursued the line pattern, speed increases that changed the apparent path of the dot when the dot was tracked had only a small effect, which was shown to result from incomplete tracking. We concluded that, in that case, the dot's apparent path was not due to induced motion, but rather to the retinal path of the dot's image.
\end{abstract}

The experiments to be reported deal with the observation that the extent of induced motion becomes smaller when the speed of the inducing motion is increased. One of our questions was directed to whether the speed effect was connected with the blurring of the moving pattern that is caused by high motion speeds or whether it was a characteristic of induced motion. The other issue of our investigation was whether or not the observation condition mattered: Does the effect of speedup take place no matter whether the subject looks at the target that goes into induced motion or whether the eyes track the moving pattern that causes the induced motion?

We first observed the speed effect in connection with an arrangement that Wallach, Bacon, and Schulman (1978) employed to measure adaptation of induced motion. ${ }^{1}$ A tall pattern of vertical lines was made to move alternately to the right and to the left, and would have caused a stationary dot, about which the moving pattern was centered, to appear to shift left and right in induced motion. But the dot was not stationary; instead, it moved upwards when the pattern moved to the right and downwards when it moved to the left. Here the induced motion manifested itself in a change in the motion path of the dot. When it moved upwards, induced motion shifted it simultaneously to the left, resulting in a sloping path, and when the pattern and the dot reversed their motions, the dot appeared to move downwards at the same slope angle. Estimates of that slope angle were used to measure the extent of the induced motion. We

This work was supported by Grant 11089 from the National Institute of Mental Health to Swarthmore College, Hans Wallach, principal investigator. The authors' mailing address is: Department of Psychology, Swarthmore College, Swarthmore, Pennsylvania 19081. noticed that speeding up the reciprocating motion of the line pattern and the simultaneous motion of the dot made the path of the dot appear less sloping. The horizontal component of the dot's path, the one that was caused by induced motion, had apparently become smaller. Because we expected it to yield more accurate measurements, the present investigation studied the speed effect with a different, but analogous, arrangement.

When physically a point is made to move simultaneously in two equal simple harmonic, reciprocating motions, for example, one horizontal and the other vertical, and with a phase shift of $90 \mathrm{deg}$, the resultant motion path is circular and is perceived as circular. Such a Lissajous path can be perceived also when only the vertical motion is objective and given by ocular pursuit, and when the horizontal component consists of induced motion, although the circular path exists then only in experience. This path is an exact analogue to the sloping path with which Wallach et al. (1978) experimented; that was also a Lissajous path, with a frequency ratio of 1 to 1 , but here the two motions were in phase. When a circular path is used and the effectiveness of horizontal induced motion is diminished, it becomes elliptical, that is, narrower than high. We measured the shape of the perceived path by obtaining estimates of its height and width. For reasons that will be presented later, we took these estimates under two observation conditions, namely, when the subject's eyes pursued the vertically moving dot and when they tracked a line of the background pattern.

\section{EQUIPMENT}

Because the apparatus used by Wallach et al. (1978) could not be speeded up sufficiently, we used a new device which projected 
both the moving dot and the vertical line pattern. The subject faced a large translucent Plexiglas screen in the dark, onto which beams from two projectors could be aimed from the rear. The beams were separately intercepted by mirrors, one attached to a vertical shaft and the other attached to a horizontal shaft, so that they could be tilted in different planes. The reflected beams were superposed by a stationary beam splitter and came to a focus in the center of the screen. Two disk cranks were mounted side by side on one shaft, which was driven by a variable-speed (shunt) motor. Each crank was connected with a linkage rod to one of the mirror shafts in such a way that the light beams reflected by the mirrors underwent reciprocating motions that were nearly simple harmonic, one in a vertical and the other in a horizontal plane. Adjustments of the cranks made it possible to set the amplitudes and the phase relations of the beam motions.

The horizontally moving beam projected a vertical line pattern on the screen, and the vertically moving beam projected a light dot $\mathbf{5} \mathrm{mm}$ across. The pattern consisted of 32 dark lines, on a light background, that were perceived as dark gray. The lines were $6 \mathrm{~mm}$ wide, the intervals were $14.5 \mathrm{~mm}$ wide, and the pattern was $64 \mathrm{~cm}$ wide and $96 \mathrm{~cm}$ high. The dot was in the center of this area. The horizontal excursion for the line pattern was $10 \mathrm{~cm}$, and the vertical excursion of the dot was $9.8 \mathrm{~cm}$. Because the mirrors whose changing slants caused these excursions were moved by cranks that were mounted on the same shaft, the excursions were of the same duration. They did, however, not start at the same time; rather, one excursion was at its midpoint when the other ended and when the reverse excursion started, providing the desired phase shift of $90 \mathrm{deg}$. This apparatus was used in all our experiments.

\section{EXPERIMENT 1 Measurements of the Speed Effect}

\section{Method}

The subjects were 14 paid undergraduates of Swarthmore College.

The motion path of the light dot was observed under two conditions. Either the subjects pursued the motion of the dot (which was actually straight and vertical) or they tracked one of the lines of the pattern. Under both conditions, the velocity of the reciprocating motions was varied and the shapes of the perceived motion paths were measured.

The subject was seated on an adjustable chair, which was raised to a point at which the subject's eyes were level with the midpoint of the dot's path. A headrest ensured that the distance between the eyes and the screen was $50 \mathrm{~cm}$. The motion display was shown briefly, and the subject was asked to describe the dot's path. It was invariably reported as circular, oval, or elliptical. The experimenter then described the two observation conditions and explained how to give estimates of the height or the width of the motion path. This was to be done by setting the gap in a pair of clearly marked calipers, one for the height and the other for the width. During the motion display, which lasted $18 \mathrm{sec}$, there was no light in the room. Afterwards, a lamp was lit so that the subject could see the calipers while making the estimates.

Seven different speeds of the motion of pattern and dot, ranging from .26 to 1.39 cycles per second (cps) were presented for each observation condition. They amounted to average speeds that ranged from 5.9 to $31.7 \mathrm{deg} / \mathrm{sec}$ for the line pattern. ${ }^{2}$ For seven subjects, the dot-pursuit condition preceded the line-tracking condition, and for the other seven, the order was reversed. The order of motion speeds was varied so that it was counterbalanced for the group as a whole.

\section{Results}

Measures of the perceived shapes of the motion paths were obtained by forming width/height ratios from each pair of estimates. Such a ratio became a subject's score for a particular combination of motion speed and observation condition. To make the distribution of these ratio scores symmetrical about 1 , scores above 1 were transformed according to the following formula: transformed ratio $=2-1 /$ ratio. The $w / h$ ratios for the two observation conditions are plotted in Figure 1. When the subject's eyes pursued the dot, the width of its perceived path narrowed to one-half as the motion speed increased about fivefold. When the pattern was tracked, the effect of speed increase was much smaller; the dot's path became narrower by $14 \%$. At the highest speed of $1.39 \mathrm{cps}$, the $\mathrm{w} / \mathrm{h}$ ratio of the motion path was .57 when the eyes pursued the dot and .92 when they tracked the lines. The difference was significant at the .0002 level $[t(13)=5.05]$. The ratios seemed to drop in a regular fashion as speed increased. Linear regression analyses on the means showed strong linear components. The ratio-speed correlation was -.99 in the dot-pursuit condition and -.96 in the linetracking condition. The average slope of the drop in the dot-pursuit condition was -.45 and in the linetracking condition, -.16 . This difference was significant at the .005 level $[t(13)=3.37]$.

\section{EXPERIMENT 2 The Cause of the Speed Effect in the Line Tracking Condition}

The question arose as to whether the small change in shape of the dot's apparent path when speed was increased in the line-tracking condition was a property of the motion process or whether it resulted from a progressive shortening of the horizontal pursuit eye movements. In our next experiment, we tested for such faulty tracking when higher motion speeds required pursuit movements to become faster.

\section{Method}

The subjects were 14 paid undergraduates.

We measured the shortfall in the pursuit of the line pattern with the afterimage method. A small point, visible as a mark on one



Figure 1. Mean width-to-height ratios of circular or oval motion path under two observation conditions, tracking of the horizontally moving line pattern or pursuit of the vertically moving dot. 
of the center lines, was added to the line-pattern slide. A photoflash with a small opening was placed at right angles to the screen on the subject's left. It was visible to the subject, who faced the screen, by reflection in a transparent mirror, that was placed between the subject's eyes and the screen and formed an angle of $45 \mathrm{deg}$ with the screen. The position of the subject's eyes was fixed by a headrest. For each subject, the photoflash was so positioned that the mirror reflection of its opening coincided with the right endpoint of the excursion of the mark that moved with the line pattern. Since the distance between the subject's eyes and this endpoint was the same as the optical distance to the opening of the photoflash, observation could be binocular. The photoflash was set off by a switch that was activated by the motion of the mirror crank that shifted the projected line pattern. The flash occurred at the moment when the reciprocating motion of the line pattern reached that endpoint where the mark coincided with the flash. When the subject's eyes tracked the shifting mark accurately, the flash would cause an afterimage on the fovea. A shortening of the reciprocating tracking movements would cause the flash to be located extrafoveally. In that case, the interval between the afterimage and the fovea would correspond to the visual angle of that shortfall. An override switch in the flash circuit permitted the experimenter to control at which motion cycle the flash would occur.

Except for the added mark, the observation conditions were the same as in the line-tracking condition of Experiment 1. The experimenter let the subject observe a number of motion cycles and then closed the switch that caused the flash to go off at the end of the motion cycle. Immediately after the flash, the subject turned his or her head to the right, rested the forehead against a bar, and fixated a mark on a white paper pad, at a distance from the eyes that was the same as the observation distance of the line pattern. When the afterimage emerged, he marked its location with a pencil.

The discrepancy between the eye position and the location of the tracked line, which the afterimage method measured, might also be caused by a falling behind of the pursuit movements in relation to the line's actual motion. Such a lagging of the eye movements in relation to the motion of the tracked target, however, would have caused a deformation of the dot's perceived motion path that was different from the one that was observed and measured in the dot-pursuit condition of Experiment 1. It would have resulted in a tilted oval path instead of merely a narrowed one. We were alert to this possibility because we were, for a different reason, prepared to find this deformation at high speeds. It never occurred under either observation condition.

Before being tested for the shortfall in the pursuit of the line pattern at a particular speed, the subject gave a width and a height estimate, as in the previous experiment. Three of the previous speeds were used, $.26, .83$, and $1.39 \mathrm{cps}$.

\section{Results}

The results are presented in Table 1 , where the $w / h$ ratios obtained from our present subjects are listed in the first row. The $w / h$ ratios that were obtained at the same speeds in the line-tracking condition of Experiment 1 are listed in the second row. At the highest speed, our present subjects showed a greater width loss, which was, however, not significant. The means of the visual angles of the distance between the afterimage location and the fixation mark, which are measures of the shortfall in pursuit, are listed in the third row. Only the highest speed produced an appreciable shortfall, and that agreed with the means of the $w / h$ ratios, which also showed a narrowing of the perceived motion path only at the highest speed. At that speed, the mean shortfall was $1.49 \mathrm{deg}$, which
Table 1

Mean Width-to-Height (w/h) Ratios of Circular or Oval Motion Path During Line Tracking and Shortening of Pursuit Movements

\begin{tabular}{lrrr}
\hline & \multicolumn{3}{c}{ Speeds (in cps) } \\
\cline { 2 - 4 } & .26 & .83 & 1.39 \\
\hline w/h Ratio (Experiment 2) & 1.14 & 1.04 & .74 \\
w/h Ratio (Experiment 1) & 1.07 & 1.01 & .92 \\
Shortfall in Pursuit (in degrees) & .18 & .36 & 1.49 \\
\hline
\end{tabular}

Note-cps $=$ cycles per second.

meant that the pursuit eye movement was shortened in the proportion of $\mathbf{. 7 3 8}$. This result was in good agreement with the mean $w / h$ ratio of .74 that was obtained from the same subjects at that speed. That the perceived shape of the motion path is closely related to the extent of the horizontal pursuit movement that the subjects' eyes performed was further demonstrated by the high correlation that obtained between the individual subjects' shortened pursuit and the $w / h$ ratio of their shape estimates. The coefficient $r$ was -.7 , which was significant at the .01 level. These results suggests a causal connection between the apparent narrowing of the dot's circular path in the line-tracking condition that was measured by the $w / h$ ratio and the shortened pursuit movements of the eyes at the high motion speed.

\section{EXPERIMENT 3 \\ Is the Speed Effect Caused by a Blurring of the Line Pattern?}

When the eyes pursue the vertically moving dot, the motion of the line pattern is given as displacement of its retinal image, and, with high motion speeds, the images of the lines get blurred. No such blurring occurs when the eyes track the pattern. Therefore, the assumption that blurring of the inducing pattern diminished the extent of induced motion would explain why speed-up diminished induced motion when the eyes remained fixed on the dot but did not do so when the line pattern was tracked, except when speed-up caused shortened pursuit eye movements.

To find out whether blurring of the line pattern diminishes the extent of induced motion, we used a strongly blurred pattern in both observation conditions. If blurring diminishes the effect of induced motion, the $w / h$ ratio should be smaller under both conditions, even at the slowest speed, and in the dot pursuit condition the decrease of the $w / h$ ratio with increasing speed should be greatly diminished, because the strong objective blurring would obscure the blurring due to the displacement of the line images. The objective blurring was achieved by focusing the projector with the line pattern slide for a distance 
of $255 \mathrm{~cm}$ while the length of the path between the projector and the screen remained $379 \mathrm{~cm}$. Under these conditions, the pattern resembled a sinusoidal spatial frequency grating of light and dark vertical bands. To enable the subject to track the blurred line pattern correctly, a slide of clear glass with a small black point at its center was added to the line pattern slide in its projector. The two slides were so spaced that the black point was in sharp focus on the screen when the line pattern was blurred. In the control conditions, in which the line pattern was sharply focused, the spacing between the slides was removed. The black point was absent during the dot pursuit condition.

\section{Method}

Twelve paid undergraduates served as subjects.

The shape of the perceived motion path was measured twice under the two viewing conditions of Experiment 1, once with the line pattern blurred and again with the line pattern sharp. Only three different motion speeds were used, $.26, .73$, and $1.20 \mathrm{cps}$. In other respects, observation and measurement conditions were the same as in Experiment 1. For both pairs of observation conditions, a subject was presented with the three speeds in fixed order. For half of the subjects, the order was from high to low, and for the other half, the order was reversed. The order of pairs of observation conditions was counterbalanced for the subject group as a whole.

\section{Results}

The 12 mean $\mathrm{w} / \mathrm{h}$ ratios that were obtained at three different speeds under two observation conditions and when the pattern was either sharp or blurred are presented in Table 2 . It is easily seen that, again, except for the slowest speed, the dot-pursuit and the line-tracking conditions produce very differently shaped paths, but that blurring the pattern made very little difference. An analysis of variance of the means yielded a highly significant effect of the observation condition $[F(1,11)=391.2, p<.001]$, but blurring had no significant effect $[F(1,11)=3.46, p=.09]$, and the interaction was not significant either. An analysis of the speed effect showed the slopes to be significantly steeper in the dot-pursuit conditions, with $b=-.49$, than in the line-tracking condition,

Table 2

Mean Width-to-Height (w/h) Ratios of Circular or Oval Motion Path Under Two Observation Conditions With Pattern of Sharp Lines or of Blurred Bands at Three Speeds

\begin{tabular}{lccc}
\hline & \multicolumn{3}{c}{ Speeds (in cps) } \\
\cline { 2 - 4 } & .26 & .73 & 1.20 \\
\hline Sharp & Horizontal Pursuit of Pattern \\
Blurred & 1.03 & .93 & .87 \\
& .99 & .96 & .83 \\
Sharp & Vertical Pursuit of Dot & \\
Blurred & .98 & .79 & .52 \\
\hline
\end{tabular}

Note-cps $=$ cycles per second. where $b$ was $-.17[F(1,11)=35.7, p<.0001]$. Blurring did not affect the slopes, and the interaction was not significant.

These results confirmed those of Experiment 1, where the slopes were very similar, -.45 and -.16 . When a blurred pattern caused the induced motion, the effect of increasing speed in the dot-pursuit condition remained undiminished. Blurring of the line pattern could not have been responsible for the strong effect of speed-up in the dot-pursuit condition. The results of this experiment are remarkable apart from the argument they yield: A maximally blurred pattern caused an induced motion that was nearly as great as that produced by a pattern of sharp lines.

Experiment 4 was undertaken to make this finding secure. It seemed necessary to show that, in Experiment 3 , induced motion was produced at least in part by the lines or by the light and dark shaded bands in the vicinity of the dot and not merely by the moving margins of the patterns. If only the margins of the patterns caused the induced motion, it would not be necessary to accept that the blurred pattern could evoke induced motion. We demonstrated the effectiveness of the patterns themselves by enlarging the visual angle they subtended to the point at which the margins were given so far in the periphery of the subject's vision that they hardly contributed to the dot's induced motion.

\section{EXPERIMENT 4 Induced Motion is Obtained Without the Lateral Margins}

Two steps were taken to enlarge the visual angle that the patterns subtended. The distance between the projectors and the screen on which the moving display appeared was increased so that the linear size of the display was $20 \%$ greater, and the observation distance was decreased to $18 \mathrm{~cm}$. As a result, the horizontal width of the pattern subtended an angle of $130 \mathrm{deg}$, and the horizontal motion of a pattern caused its vertical margins to shift between positions that were 61 and $68 \mathrm{deg}$ to each side of the center of the display. It had been found that margin motions at such a large distance from the dot had little effect on its apparent motion.

\section{Method}

Seven paid undergraduates were used as subjects.

Three displays were used. In addition to the line pattern and the blurred pattern that were used in the previous experiment, there was one display in which the margins of an empty slide frame were projected in place of the line-pattern slide. W/h estimates were obtained at the three speeds that were used in Experiment 3, but only for the dot-pursuit condition. Instead of the horizontal tracking condition, each subject went through the entire experiment once more; eventually each score was the average of two $w / h$ estimates. The order of presentation of the three displays was counterbalanced across six subjects. Four subjects received the speed conditions in sequence from low to high and three in 
the opposite order. For every subject, all presentation orders were reversed for the second set of estimates.

\section{Results}

Table 3 shows the mean $w / h$ estimates for the three display conditions. As in the dot-pursuit condition of Experiment 3, the width of the perceived motion path narrowed as motion speeds increased for both the sharp and the blurred pattern. The slopes of the mean $w / h$ ratios were significant at the .003 and .01 level, respectively. The slope for the line pattern, with $b=-.39$, was not very different from the corresponding slope in Experiment $1(\mathrm{~b}=-.45)$. For the blurred pattern, the slope was somewhat less, namely, $b=-.32$, but the difference was not significant. The $w / h$ ratios themselves were, however, smaller than in the previous experiments. This was probably due to the larger visual angle used in the displays. The larger projections had two effects. The angular motion speed in the vicinity of the dot was greater by a factor of 2.8 , and if the speed effect is related to the rate of the angular displacement of the moving pattern, this increase in the angular motion speed would account for about half the difference between the results of the two experiments. ${ }^{3}$ Secondly, the large projection of the display increased the distances between the lines of the pattern in the vicinity of

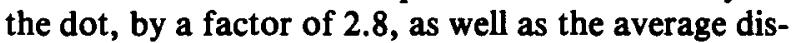
tance between the dot and the lines, the landmarks that produced most of the induced motion as it turned out. Such diminished density of the inducing pattern diminishes induced motion. Although the blurred and the line pattern in Experiment 3 produced induced motion of approximately the same extent, the averaged $w / h$ ratios for the blurred pattern were significantly smaller than those for the line pattern $[t(12)$ $=2.72, p<.02]$, namely, .53 vs. .64 . The diminished induced motion which the blurred pattern caused here was probably due to the increased visual angle of the cycle of light and dark shaded bands, which changed the slope of the shifting brightness gradients in the vicinity of the dot by a factor of $1 / 2.8$.

The patternless condition yielded very little induced motion. The $w / h$ ratios were not above .1 . Thus, only a very small part of the effect of the pattern motion can be ascribed to the motions of its

Table 3

Mean Width-to Height (w/h) Ratios of Motion Path During Pursuit of the Vertically Moving Dot for Three Displays at Three Speeds

\begin{tabular}{lccc}
\hline & \multicolumn{3}{c}{ Speeds (in cps) } \\
\cline { 2 - 4 } \multicolumn{1}{c}{ Displays } & .26 & .73 & 1.20 \\
\hline Sharp Lines & .82 & .66 & .45 \\
Blurred Bands & .64 & .57 & .36 \\
Blank Field & .08 & .10 & .08 \\
\hline
\end{tabular}

Note-cps $=$ cycles per seconds. margins. Most of the induced motion resulted from the patterns themselves. A maximally blurred pattern produced an induced motion measured by a $\mathrm{w} / \mathrm{h}$ ratio of .64 , at the slowest speed, of which .56 $(.64-.08)$ was attributable to the pattern itself.

\section{DISCUSSION}

Speed-up greatly diminished induced motion when the eyes tracked the vertically moving dot, but had a much smaller effect when the eyes tracked the line pattern. Blurring the line pattern did not alter these results significantly, except when the cycle of the blurred pattern subtended a visual angle larger than about 1 deg. Experiment 2 led to the conclusion that the small speed effect that occurred in the line-tracking condition resulted from shortened pursuit eye movements.

Experiment 4 also throws light on another issue. To what degree do changes in egocentric localization, the Roelofs effect, contribute to induced motion that is caused by a moving pattern? Wallach, O'Leary, and McMahon (1982) had found that replacing the moving margins of a line pattern similar to ours with fixed panel edges diminished induced motion by nearly $30 \%$, but how much of this change was caused by the stationary panel edges, which now served as contradictory landmarks, and how much was due to absence of the Roelofs effect remained, of course, unknown. The strongly peripheral location of the moving margins in our blank field condition greatly diminished their landmark effect. That this nearly abolished induced motion showed that the Roelofs effect played a very minor role in the experiments under discussion. ${ }^{4}$

Our remaining concern is with the conclusion that there was no speed effect in the line-tracking condition. Ours is not the only instance in which a factor that diminished induced motion did so only when the eyes tracked the target that underwent induced motion but failed to do so when the eyes pursued the surrounding line pattern. When Wallach et al. (1978) measured adaptation to induced motion, which also diminished the extent of induced motion, they, too, found that the effect became manifest when the subject's eyes tracked the target that underwent induced motion but failed to do so when the line pattern was tracked. (As mentioned earlier, Wallach et al. (1978) employed a display that resembled ours, but omitted the 90-deg phase shift, so that induced motion caused the vertically moving target to move on a sloping straight path.) The authors considered two explanations for this result: One assumed that the effect of adaptation was tied to the viewing condition that was employed in the exposure that caused the adaptation and, therefore, failed to become manifest when the line pattern was tracked. The other explanation was 
more radical. It assumed that the sloping path of the vertically moving target that was perceived during line tracking did not result from induced motion, but from the oblique displacement of the retinal image with which the vertically moving target is given when the eyes are making simultaneous horizontal pursuit movements. The perceived path could, therefore, not show the adaptation effect. This explanation is supported by the results of our investigation, which showed that diminished induced motion that had an entirely different and, as yet, unknown cause did not become manifest during line tracking either. Except for incomplete ocular pursuit of the line pattern, line tracking here caused a circular path of the dot's retinal image, and such a path was perceived. That such different antecedent conditions produced parallel results argues that they operate in the same way and that that is the case when image path perception takes place.

This argument is supported by the close relation we found in Experiment 2 between the perceived shape of the dot's motion path and the shortened pursuit eye movements that were measured in the line-tracking condition at the highest speed. Since the shortened tracking movements cause a narrowing of the motion path of the dot's image on the retina, this relation makes sense if the image path is directly perceived. The proposition that the retinal path of the dot's image leads directly to its perceived motion when the moving surround of the dot is tracked will be taken up in a future publication.

\section{REFERENCES}

Goces, W. C. Induced motion as a function of the speed of the inducing object, measured by means of two methods. Perception, 1979, 8, 255-262.

Wallach, H., Bacon, J., \& Schulman, P. Adaptation in motion perception: Alteration of induced motion. Perception \& Psychophysics, 1978, 24, 509-514.

Wallach, H., O'Leary, A., \& McMahon, M. L. Three stimuli for visual motion compared. Perception \& Psychophysics, 1982, 32, 1-6.

\section{NOTES}

1. Gogel (1979) also was concerned with speed of induced motion, but for different reasons. The velocities he employed were much smaller than those that produced the speed effect we investigated.

2. The average speeds are for whole excursions. Because the presented motions were simple harmonic, the speeds at the center half of the excursion were 1.5 times as high.

3. We arrived at this estimate by multiplying the slowest speed of $.26 \mathrm{cps}$ by 2.8 and by obtaining an interpolated value of the $\mathrm{w} / \mathrm{h}$ ratio at that speed from the results of Experiment 1.

4. There is no reason to assume that peripheral location of the margins interferes with the Roelofs effect. On the contrary, egocentric localization is supposed to be strongly influenced by peripheral vision.

(Manuscript received January 28, 1983; revision accepted for publication May 24,1983 .) 